\title{
Hypothèse de parcours diagnostique du défi- cit érectile à la lumière de connaissances récentes sur l'anatomo-physiologie de l'érec- tion et sur la thérapie des troubles érectiles
}

\author{
G. PASSAVANTI , V. PIZZUTI , R. PAOLINI \\ O. Urologie-Andrologie Ospedale "Misericordia"Via senese 58100 Grosseto (Italie)
}

\section{RÉSUMÉ}

Actuellement le bilan diagnostique du déficit érectile comprend un large spectre d'examens; chaque test donne des informations spécifiques sur la pathogenèse du syndrome étudié, mais en même temps a des limitations importantes pour poser un diagnostic étiologique. Le déficit érectile doit être considéré comme un syndrome multifactoriel dans lequel les composantes organique et psychogène coexistent en proportion variable. L'examen de 66 cas cliniques nous permet d'une façon empirique, de faire une corrélation entre les donnés de la rigidomètrie nocturne et les données de l'ECCD et de la PHCM.

Nous pouvons relever que la rigidométrie ne permet pas de faire un diagnostic différentiel entre les troubles organiques et psychogènes de l'érection; elle ne permet pas non plus de prévoir les résultats de la thérapie ; l'intégration des résultats des différents examens permet de définir la pathogenèse du déficit érectile, mais ne permet pas d'identifier l'étiologie. Donc le but de la prise en charge du patient avec des troubles de l'érection est de résoudre le symptôme et satisfaire ses attentes. On peut proposer un parcours dia- gnostique partagé sur deux niveaux : le premier qui comprend l'anamnèse, l'examen objectif, l'injection intracaverneuse de PGE 1 , le bilan hormonal et la rigidomètrie, le second qui comprend des examens plus complexes (ECCD, PHCM,) et doit être entrepris quand on n'a pas réussi à résoudre la trouble érectile ou quand on envisage une thérapeutique chirurgicale.

Mots clés : Démarche diagnostique du déficit érectile, rigidomètrie nocturne

\section{INTRODUCTION}

Le parcours diagnostique du déficit érectile a été modifié pendant ces dernières années à cause de l'évolution des techniques d'imagerie, des connaissances sur l'anatomie des corps caverneux et sur la physiologie de l'érection et des nouvelles thérapies. Nous sommes partis de notre expérience pratique pour proposer, d'une façon empirique, un parcours et une méthode diagnostique du déficit érectile. 


\section{MATÉRIEL ET MÉTHODE}

66 patients, âge compris entre 44 et 71 ans, ont été étudiés pour un trouble de l'érection. Ils ont eu une anamnèse et un examen objectif approfondi ; une rigidométrie (RIGISCAN) nocturne, un echo-couleur-doppler dynamique (ECCD) et, 53 sujets ont eu une pharmacocavernosométrie (PHCM).

Le RIGISCAN a été exécuté pendant 3 nuits, et nous avons considéré pathologiques une rigidité inférieure à $60 \%$ et une durée de chaque phase érectile inférieure à $10 \mathrm{~min}$.

L'echo-couleur-doppler (ECCD) était éxecuté après une injection intracaverneuse de $20 \mathrm{mcg}$ d'alprostadil et avec un "stimulus videosexuel" (VSS). Il y avait un déficit artériel si la Vitesse Sistolique (VS) était inférieure à $25 \mathrm{~cm} / \mathrm{sec}$; il y avait une incontinence cavernoveineuse si la Vitesse Diastolique (VD) était supérieure à $8 \mathrm{~cm} / \mathrm{sec}$ et si l'IR (Resistence Index) inférieur des $80 \%$.

La pharmacocavernosométrie (PHCM) était executée après injection de $20 \mathrm{mcg}$ d'alprostadil et avec VSS, elle était positive si le flux minimum était supérieur à $20 \mathrm{ml} / \mathrm{min}$ et si la chute de la pression intracaverneuse était supérieure à $30 \mathrm{mmhg} / 30 \mathrm{sec}$.

\section{RESULTATS}

$13(19,7 \%)$ patients avaient un RIGISCAN avec des phases érectiles d'une rigidité $<60 \%$ et d'une durée $<10 \mathrm{~min}$. L'ECCD relevait un déficit érectile et une VS $<25 \mathrm{~cm} / \mathrm{sec}$.

$25(37,88 \%)$ patients avaient un Rigiscan pathologique (10 avaient une rigidité comprise entre 50 et $60 \%$ et une durée $<10 \mathrm{~min}, 15$ avaient une rigidité $<50 \%$ et une durée $<10$ min). Ils avaient tous, à l'ECCD, une VD $>8$ $\mathrm{cm} / \mathrm{sec}$., un IR $<0,8$ et la $\mathrm{PHCM}$ relevait une incontinence caverno-veineuse.

$17(25,76 \%)$ patients avaient une rigidité $<60$ $\%$ et une durée $<10 \mathrm{~min}$; l'ECCD et la PHCM ne démontraient pas un déficit érectile après injection intracaverneuse

$8(12,12 \%)$ patients avaient un RIGISCAN normal et ECCD et PHCM négatifs pour un déficit érectile après stimulation pharmacologique.
$3(4,55 \%)$ patients avaient une rigidité $<60 \%$ et durée $>10$ min., mais l'ECCD et la PHCM relevaient un déficit des mécanismes venoocclusifs ( hyper-tonus $\alpha$ ?).

Les 13 patients avec un déficit artériel ont été soignés par des injections intracaverneuses d'alprostadil avec des résultats insatisfaisants et donc chez 2 patients ont été placées des prothèses ; les 53 patients restants sont en thérapie avec l'alprostadil intracaverneux à la dose de 20- 30 mcg.(Fig.1)

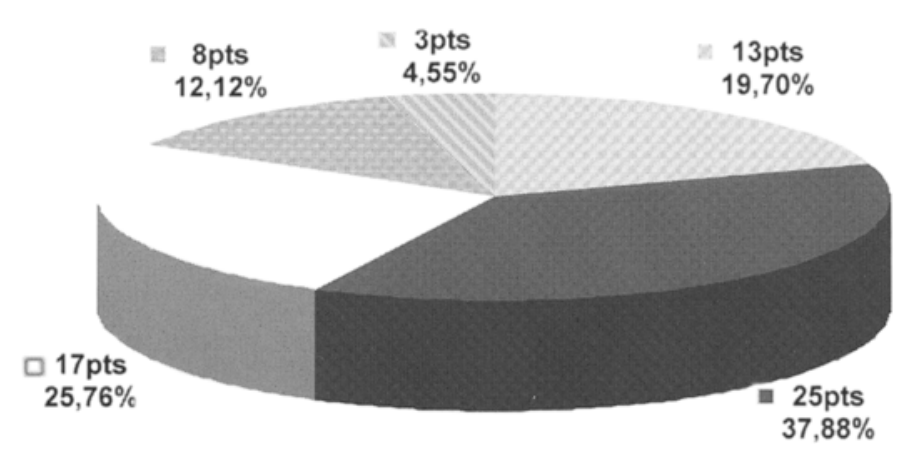

Figure1 : Distribution des patients (pts) selon les résultats de RIGISCAN, ECCD, PHCM

- 13 pts rigidité<60\%, durée< $<10 \mathrm{~min}$. $V S<25 \mathrm{~cm} / \mathrm{sec}$.

- 25 pts Rigiscan pathologique, $V D>8 \mathrm{~cm} / \mathrm{sec}$. Incontinence caverno-veineuse

- 17 pts rigidité< $60 \%$, durée $<10$ min. Absence de déficit érectile.

- 8 pts Rigiscan. ECCD, PHCM negatifs pour déficit érectile

- 3 pts rigidité $<60 \%$, durée $>10$ min. Incontinence caverno-veineuse (hyper-tonus $\alpha$ )

\section{DISCUSSION}

La démarche diagnostique du déficit érectile considère : une anamnèse scrupuleuse,un examen objectif, une rigidométrie nocturne ou en temps réél, l'ECCD et la PHCM avec des test provocatifs. Ces examens ont tous des avantages et des limites désormais bien connus. Les avantages et les limites du RIGISCAN sont très intéressants; au début on pensait que la rigidomètrie aurait permis le diagnostique différentiel entre déficit érectile organique et psy- 
chogène [9], mais ça n'a été pas obtenu [16], parce que le RIGISCAN enregistre la rigidité circulaire et non pas la rigidité axiale du pénis [1].

L'ECCD dynamique avec des tests provocatifs (Redosing, VSS, Squeezing) évalue d'une façon très précise la circulation artérielle des corps caverneux $[11,15]$ et il donne aussi une évaluation approximative de la continence caverno-veineuse avec les mesures de la VD et de l'IR $[3,8]$; cependant il ne donne pas des renseignements précis sur la géométrie des corps caverneux et sur le relâchement du tissu musculaire lisse des sinusoïdes [4]. Le point de repère pour le diagnostic d'incontinence caverno-veineuse est la $\mathrm{PHCM}$ avec test provocatifs [14]. La PHCM est soumise à l'état psychoémotif du sujet et donc les tests provocatifs sont essentiels pour obtenir le maximum de relâchement possible [6]. La pharmacocavernosographie est peu utile [12].

La composante psycho-émotive joue un rôle peu clair dans l'étiologie du déficit érectile; elle peut être déterminée par une inhibition des centres hypothalamiques qui entraîne une inhibition des centres médullaires parasympathiques et une excitation du tonus $\alpha$-adrénergique $[5,17]$. Ces mécanismes déterminent un déficit du relâchement musculaire lisse. Selon l'importance de ce phénomène on pourra avoir, parfois une absence d'érection et parfois une érection instable $[6,10]$. Les tests provocatifs (Redosing, VSS, Squeezing) ont été proposés pour réduire l'interférence déterminée par le susdit phénomène [6].

L'évaluation de nos donnés cliniques nous a permis de proposer des réflexions empiriques, mais significatives:

-La rigidomètrie nocturne n'a jamais permis d'opérer un diagnostic différentiel précis entre patients avec un déficit érectile organique ou psychogène et elle n'a jamais permis de prévoir la réponse à la thérapie.

-L'intégration de plusieurs tests diagnostiques ne permet pas d'identifier l'étiologie du déficit érectile, mais elle permet de préciser la pathogenèse : le déficit artériel et/ou surtout, l'incontinence caverno-veineuse.

Le déficit érectile, en général, est l'expression de plusieurs facteurs étiologiques [2] lesquels causent un syndrome dont la trouble de l'érection est souvent l'aspect principal [13]. Les deux composantes, organique et psychogène, ne sont jamais séparées et partagées, mais elles coexistent et l'une ou l'autre peut parfois prévaloir [10].

Sur ces bases le but de la prise en charge du déficit érectile, à la lumière de récentes connaissances sur l'anatomo-physiologie de l'érection, sur la pathogenèse du déficit érectile et sur les nombreuses possibilités thérapeutiques, est le patient's goal directed therapy [7].

Donc, si l'étude du sujet avec un trouble de l'érection a le but de satisfaire les besoins du patient, nous proposons un parcours diagnostique partagé sur deux niveaux :

Le premier comprend l'anamnèse, l'examen objectif, l'éventuel bilan hormonal, l'injection intracaverneuse de PGE1 et la rigidomètrie. Lorsqu'on ne réussit pas à résoudre le symptôme du patient ou, quand on envisage une thérapeutique chirurgicale on passe au deuxième niveau qui comprend l'ECCD et la PHCM. Il faut réserver les examens plus invasifs ou complexes (NMR,EMG,FCG, Scintigraphie dynamique) à des patients sélectionnés sur leur pathologie (ex. Maladie de La Peyronie).

\section{CONCLUSION}

Nos réflexions nous permettent de souligner que le parcours diagnostique du déficit érectile est changé d'une façon évidente à cause du progrès des connaissances sur l'anatomie des corps caverneux, sur la physiologie de l'érection, la physiopathologie des troubles de l'érection, les développements de la technologie appliquée à l'imagerie et des progrès thérapeutiques. Néanmoins, puisque le déficit érectile est l'expression de tableaux nosologiques et étiologiques complexes, le but de la prise en charge du patient, est de résoudre le symptôme.

Le parcours diagnostique peut être partagé sur deux niveaux dont un qui comprend des tests diagnostiques de base peu invasifs, et le deuxième qu'il faut appliquer quand on n'a pas réussi à résoudre le symptôme, ou quand on envisage une possibilité chirurgicale, et qui comprend des examens plus complexes et 
invasifs.

\section{RÉFÉRENCES}

1. ALLEN R., SMOLEV J., ENGEL R., BRENDLER C. Comparison of rigiscan and formal nocturnal penile tumescence testing in the evaluation of erectile rigidity Urol. 1993, $149: 1265-1268$.

2. BORTOLOTTI A., PARAZZINI F., COLLI E ., LANDONI M. : The epidemiology of erectile dysfunction and its risk factors Intern. J. Androl. 1997, 20 : 323-334.

3. De MEYER J., THIBO P. : The correlation among cavernous presure, penile rigidity and resistance index Urol. 1998, $160: 63-66$.

4. De MEYER J., THIBO P. : The effect of re-dosing of vasodilators on the intracavernosal pressure on the penile rigidity Eur. Urol. 1998, 33 : 293-297.

5. GIULIANO F., RAMPIN O., BENOIT G., JARDIN A. : Pharmacologie périphérique de l'érection Progr. Urol. 1997, : 24-33.

6. HATZICHRISTOU D., SAENZ DE TEJADA I., NAMBURI S., GOLDSTEIN I. : In vivo assessment of trabecular smooth muscle tone, its application in pharmaco -cavernosometry and analysis of intracavernous pressure determinants Urol. 1995, $153: 1126-1135$.

7. LUE T. F. : Impotence : a patient's goal-directed approach to treatment World J. Urol. 1990, $8: 67-74$.

8. MERCKX L., De BRUYNE R., GOES E., DERDE M., KEUPPENS F. : The value of dynamic color duplex scanning in the diagnosis of venogenic impotence J.Urol. 1992, $148:$ 318-320.

9. MORALES A., CONDRA M., REYD K. : The role of nocturnal penile tumescence monitoring in the diagnosis of impotence :a review J.Urol. 1990,143:441-445.

10. MONTAGUE D.K. : Erectile dysfunction : how much testing is needed in the era of effective oral pharmacotherapy? AUA 94th annual meeting 1999

11. PACELLI W., DEL GUERCIO M., LOMBARDO M., GUGLIELMELLI M. : Ruolo dell'ecocolordoppler nella diagnosi dellimpotenza di tipo venogenico Minerva angiol. 1995, $20: 465-468$.

12. PASSAVANTI G., PIZZUTI V., BUONAVIA A., SPINOSA E., PAOLINI R. : La farmacocavernosometria come test diagnostico funzionale della fuga venosa Urologia 1995, 62 : 386-390.

13. PASSAVANTI G. Le concept d'incontinence cavernoveineuse à la lumière des connaissances récentes sur l'anatomie des corps caverneux et sur la physiologie de l'érection Memoire pour le D.U. d'Andrologie "Faculté St. Antoine - Université P. et M. Curie Paris VI" 1997/98 pag.46-48,

14. PESCATORI E., ARTIBANI W. : The role of pharmacocavernosometry in the diagnostic work-up of erectile dysfunction European Urology up-date series 1996, 5 : 149-157.

15. SARTESCHI L., PAOLI R., TURCHI P., MENCHINI FABRIS G. : ECD power imaging X Congr. Naz. S.I.A. 1996,pag 3-14.

16. STAERMAN F., COEURDACIER P., GUIRAUD P., CIPOLLA B., LOBEL B. : Valeur diagnostique de l'en- registrement des érections nocturnes Progr. Urol. 1996, $6: 403-408$.

17. STOLERU S., GREGOIRE M., DECETY J. Et al. : Neurophysiologie centrale de l'érection Andrologie 1997, $7: 344-345$.

\section{ABSTRACT}

Diagnostic protocol of the erectile dysfunction : a proposal based on up-to date anatomical and physiological knowledges of the erection and on therapy of the erectile failure

$$
\text { G. PASSAVANTI, V. PIZZUTI, R PAOLINI }
$$

Nowadays the diagnosis of erectile disfunction is based on a wide range of clinical and instrumental investigations in order to obtain specific and detailed information on the pathogenesis of this syndrome, but at same time these investigations are useless to know much about the aetiology. Therefore the erectile failure must be related to organic and psychological causes always detected in each patient.

Sixty-six men complaining of erectile failure were evaluated with nocturnal penile activity registration (NPT), echo-color-doppler (ECD) and dynamic infusion cavernosometry (FCM) and the reports were, even empirically, compared.

This study pointed-out that the nocturnal rigidity is not reliable either to differentiate between erection trouble having a psychogenic or organic origin, or in anticipating the therapeutic response. Furthermore, the records of instrumental investigations (NPT,ECD,FMC) failed to point-out the aetiology of erectile failure.

Therefore, in our opinion, the main goal of the andrologist should be the prompt control of the symptom and we propose an early assessment of the patient by means of medical history, physical examination, hormonal assays, intracavernous injection of vasoactive drug, and nocturnal activity monitoring. Further investigations, including ECD and FCM, should be performed in non respondent patients or when surgical therapy is indicated.

Key Words : Diagnostic protocol of the erectile dysfunction, nocturnal rigidometry 